\title{
Shock Models Revisited
}

\author{
Malcolm Walmsley ${ }^{1}$, Guillaume Pineau des Forêts ${ }^{2}$, \\ and David Flower ${ }^{3}$ \\ ${ }^{1}$ INAF-Osservatorio Astrofisico di Arcetri, Largo E.Fermi 5, I-50125 Italy \\ email:walmsley@arcetri.astro.it \\ ${ }^{2}$ IAS, Université de Paris-Sud, 92405 Orsay Cedex, France \\ ${ }^{3}$ Physics Department, The University, Durham DH1 3LE, UK
}

\begin{abstract}
We review progress in the area of the modelling of shocks in molecular clouds. In particular, we consider what has been learnt about shock structure evolution in situations where the steady-state assumption is no longer valid. We discuss the interpretation of the observed water abundance from SWAS, Odin, and ISO. We also consider the erosion of grains in shocks as well as the effect of the presence of grains on shock structure.
\end{abstract}

\section{Introduction}

Molecular clouds exhibit supersonic linewidths and hence one might expect that their structure and chemical make-up is influenced by shocks. Shock waves cause compression and heating of the pre-shock material. Both effects have consequences for cloud structure as well as for the observed molecular abundance distribution in molecular clouds as shown in numerous articles over the past three decades (e.g., Hollenbach \& McKee 1979; McKee \& Hollenbach 1980; Draine et al. 1983; Neufeld \& Dalgarno 1989; Hollenbach 1997). In fact, the very origin of molecular clouds is likely due to shock compression (Bergin et al. 2004) and so shock chemistry may have considerable relevance for star formation and galactic evolution.

However, in this brief review, we will confine ourselves to a few topics which have attracted attention during the past 5 years due to either observational or theoretical developments. One of these is the consequences for shock structure and chemistry of departures from steady-state. We examine these in $\S 2$ and consider the observational predictions of evolution in shock structure. In $\S 3$, we consider what we have learnt from recent satellite observations of water emission of shocks and in $\S 4$, the behavior of dust grains in shocks (as well as their effect on shock structure). Finally, in $\S 5$, we discuss future directions.

\section{Non Steady Shocks}

The timescale on which matter flows through a C-shock is relatively long (dependent on the ionization degree of the pre-shock medium) and can be of the same order (a few thousand years) as estimated ages for outflows from YSOs. Thus, it is possible (see Chièze et al. 1998; Flower et al. 2003a; Lesaffre et al. 2004a; Lesaffre et al. 2004b) that the shock structures in outflow sources are evolving giving rise to a "mixed" situation where a J-type (or Jump) discontinuity is embedded in a C-type (or Continuous) flow. To complicate matters still further, the details of the chemistry and in particular the balance between atomic and molecular hydrogen influences the structure. Last, but not least, grains can considerably influence shock structure (see discussion in $\S 4$ ). 

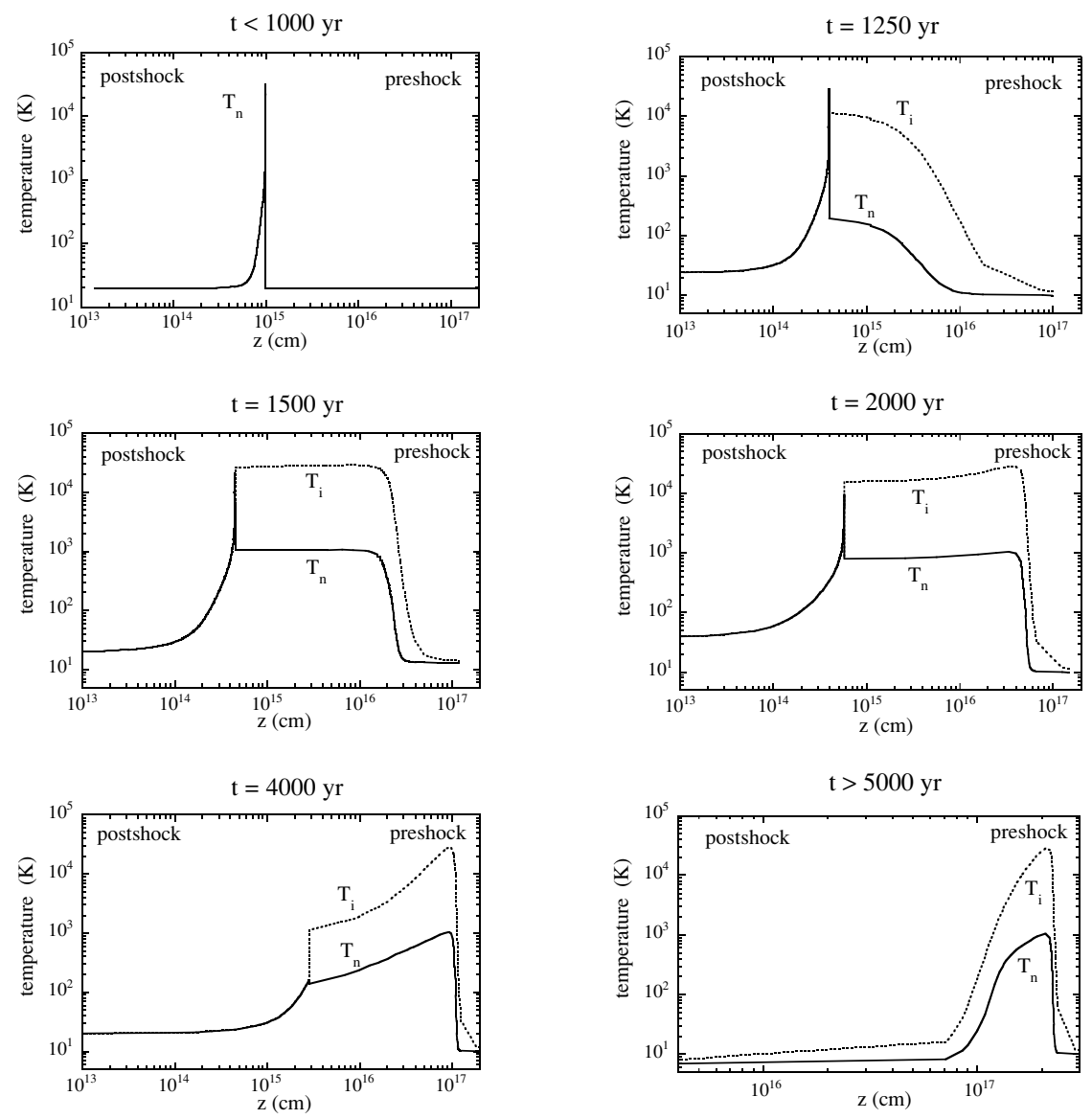

Figure 1. Computed temperature structure of C-J shock as function of time for a $25 \mathrm{~km} \mathrm{~s}^{-1}$ shock incident on a medium of density $10^{4} \mathrm{~cm}^{-3}$ and magnetic field $100 \mu \mathrm{G}$. At early times (top left), the temperature shows a J-type discontinuity with rapid cooling in the post-shock gas whereas beyond 1000 years, the shock precursor heats both ions (dotted curve) and neutrals (full curve). Beyond 5000 years (bottom right), the shock has become stationary C-type. From Pineau des Forêts \& Flower (2000).

An example showing the evolution of shock structure with time is given in Figure 1. One sees that with time, a discontinuous J-type structure evolves into a C-shock. How in detail this happens depends on both ionization degree and magnetic field among other things. One concludes that many observed outflows could be in the latter phase of this evolution. Are there observational discriminants which mark a C-J shock of this type? In fact, ISO observations of the rotational $\mathrm{H}_{2}$ lines towards some outflows do need a mixture of $\mathrm{C}$ and $\mathrm{J}$ to explain them. Figure 2 from the work of Flower et al. 2003b (see also Cabrit et al. 2004) is an example of this. One sees that whereas a C-shock fits the low-excitation lines well and a J-shock fits the high-excitation lines, one needs a C-J model to fit both.

We note, however, that these models are one dimensional and that a proper treatment of a jet-outflow system will presumably involve a variety of shocks of differing obliquities and velocities. Distinguishing between different models will require consideration of line 


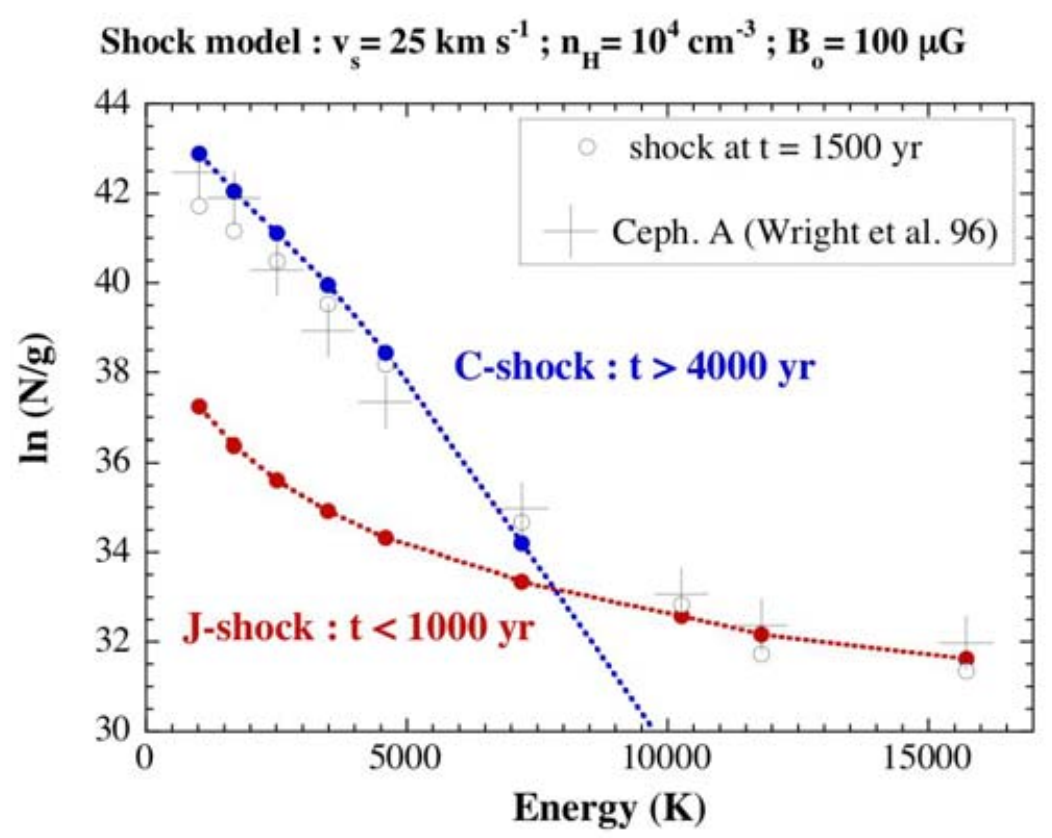

Figure 2. Log of level column density against level excitation of $\mathrm{H}_{2}$ rotational transitions for a C-J shock model with pre-shock $\mathrm{H}$ number density $10^{4} \mathrm{~cm}^{-3}$ and magnetic field $100 \mu \mathrm{G}$. The empty circles show model results for a "shock age" of 1500 years while the crosses show observations from Wright et al. (1996). The dotted lines connecting filled circles show model expectations both for a C-shock (fitting the low-excitation data) and a J-shock (fitting high-excitation lines).

profiles at well as fine scale spatial information. It will also require consideration of species such as water whose abundance varies dramatically in different contexts.

\section{The Water Conundrum}

It has been clear for many years that interstellar water plays a central role in the oxygen balance of molecular clouds. This is demonstrated by the fact that in solid form, it is often extremely abundant (see e.g., van Dishoeck 2004). Moreover, it has been known for a long time that in post-shock gas, above about $250 \mathrm{~K}$, all available oxygen gets converted into water and that for shock velocities above $25 \mathrm{~km} \mathrm{~s}^{-1}$, ice mantles become eroded (Draine et al. 1983; Flower and Pineau des Forêts 1994). Thus much shocked gas should be water rich and the searches for gas-phase water with ISO, SWAS, and Odin were based upon this assumption. The observations however only partially confirmed the theoretical predictions (though see Harwit et al. 1998, Wright et al. 2000) and in general, the water abundance was lower than expected (e.g., Snell et al. 2005; Liseau et al. 1996; Neufeld et al. 2000; Benedettini et al. 2003; González-Alfonso et al. 2002; Olofsson et al. 2003). We briefly consider in this section measurements of the water abundance in shocked regions and their interpretation.

One of the problems interpreting the water observations is that observed intensities are very sensitive to the distribution of density and temperature of the observed structures which are typically not resolved by the beams of, e.g., ISO and SWAS. This can easily cause order of magnitude uncertainty in the inferred water abundances, although one can improve matters using multi-line studies. It is also true that using $\mathrm{CO}$ as a surrogate for 
the $\mathrm{H}_{2}$ column density can be very misleading due to its differing excitation characteristics. Nevertheless, there are cases such as the interaction of the supernova remnant IC443 with surrounding molecular gas (Snell et al. 2005) where the water under-abundance is presently a challenge to the modellers. Here, one is faced with the problem not only of fitting the water data but also $\mathrm{OH}$ and atomic oxygen (Burton et al. 1990).

There are a variety of possible solutions that have been proposed to resolve the "water conundrum" but no single solution appears to pass all observational tests. In unshocked dense gas, it is possible that a large fraction of oxygen is in the form of water ice and hence is unavailable to form gas-phase water (or $\mathrm{OH}, \mathrm{O}_{2}$ etc). This can also be important in low-velocity shocked gas (below $25 \mathrm{~km} \mathrm{~s}^{-1}$ ) where sputtering is not sufficiently efficient to desorb the outflows. But the velocities detected in regions such as the IC443 clumps are much higher than this suggesting that sputtering of ice mantles is likely. It is also the case that the most suitable venue for producing hot gas-phase water in in C-shocks of, say, 20-40 $\mathrm{km} \mathrm{s}^{-1}$. These however may be suppressed if the ionization degree is much higher than that obtained assuming cosmic rays to be the main ionizing agents. Could that be true? It is not clear but certainly direct estimates of ionization degree are difficult and the question of the influence of shock produced UV is important (e.g., Sternberg \& Dalgarno 1989; Viti et al. 2003).

\section{Grains and Their Consequences}

The presence of grains influences shock structure and the destruction of grains in shocks has observational consequences. The first question has had considerable study over the past few years (Ciolek \& Roberge 2002; Ciolek et al. 2004; Flower \& Pineau des Forêts 2003) but it seems likely that the last word has not been said. Results are very sensitive to the grain mass distribution and thus, to the extent to which grains have coagulated and acquired ice mantles. Figure 3 gives a recent estimate of the boundary between $\mathrm{C}$ and J-shocks. We see that at low densities, the critical speed for $\mathrm{H}_{2}$ dissociation (Le Bourlot et al. 2002) is the dominant effect whereas at high pre-shock densities, the effective magnetosonic speed of the grains is most important (Cabrit et al. 2004).

Linked to this question is that of grain survival in shocks (see review of Jones 2000). One of the interests here is that some grains survive at shock velocities well above $100 \mathrm{~km} \mathrm{~s}^{-1}$. On the other hand, some erosion does occur in shocks with velocities of order $50-80 \mathrm{~km} \mathrm{~s}^{-1}$ as evidenced by the iron abundance measured along jets from young stars (30\% in the gas phase; Nisini et al. 2002; Nisini et al. 2005). Moreover, there is also evidence for dust emission (Smith et al. 2005) from some illuminated jets and there appear to be good prospects for comparing these results with theory. In the jets, one is presumably dealing with J-type shocks but there is also evidence for dust erosion in C-type shocks. This mainly comes from the measurements of mm-wavelength $\mathrm{SiO}$ lines (e.g., Jiménez-Serra et al. 2005) where one observes greatly enhanced SiO abundances (factor 1000) in the outflow from some young Class 0 objects. One of the peculiarities here is that one does not observe evidence for erosion of refractory elements other than Si (suggesting perhaps that silicon oxide in some form is a minor component of the dust which can be eroded in C-shocks). FeO , for example (Walmsley et al. 2002), has been searched for in many outflows but only found in the mysterious absorbing layer towards SgrB2. 


\section{$\mathbf{B}(\mu \mathbf{G})=\left[\mathbf{n}_{\mathbf{H}}\left(\mathrm{cm}^{-3}\right)\right]^{0.5}$}

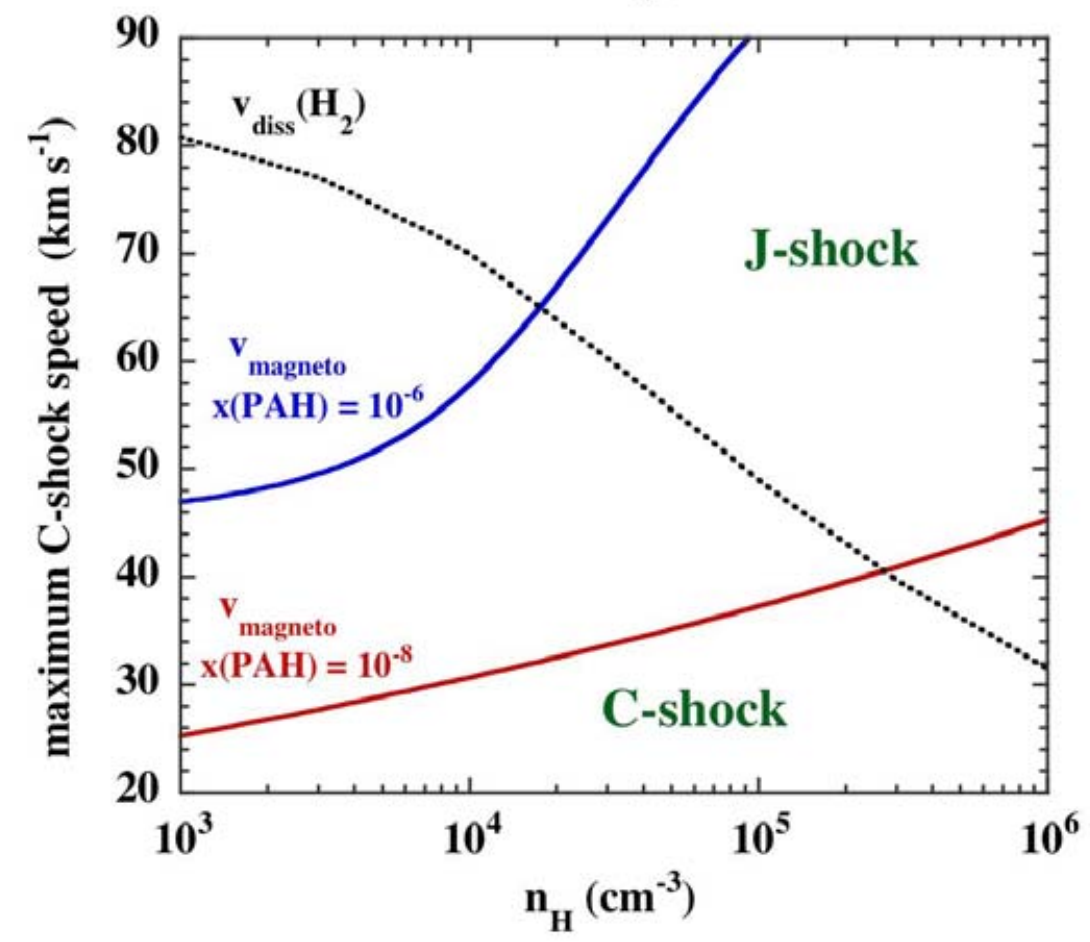

Figure 3. Critical velocity for the transition between a C-shock and a J-shock as a function of pre-shock $\mathrm{H}$ number density. The full lines show the magnetosonic speed taking grain coupling into account for two assumed abundances for PAHs. The dotted line shows the critical shock velocity for $\mathrm{H}_{2}$ dissociation from Le Bourlot et al. (2002).

\section{Future Prospects}

Clearly, the new generation of instruments is going to give new impetus to this field and some of the topics discussed here already demonstrate that. A tenth of an arc second is $50 \mathrm{AU}$ at 500 parsec (the distance of Orion). Studies at this resolution would allow one to probe the details of $\mathrm{C}$ shocks and thereby test current models with much more confidence. It is also worth noting that the increasing sophistication of MHD models may allow the insertion of chemistry and grain characteristics in a self-consistent fashion into shock models.

\section{References}

Benedettini, M., Viti, S., Giannini, T., Nisini, B., Goldsmith, P.F., \& Saraceno, P. 2003, in Chemistry as a Diagnostic of Star Formation, eds. C.L. Curry \& M. Fich (NRC Research Press), p. 248

Bergin, E.A., Hartmann, L.W., Raymond, J.C., \& Ballesteros-Paredes J. 2004, Ap. J. 612, 921 Burton, M.G., Hollenbach, D.J., Haas, M.R., \& Erickson, E.F. 1990, Ap. J. 355, 197

Cabrit, S., Flower, D.R., Pineau des Forêts, G., Le Bourlot, J., \& Ceccarelli, C. 2004, Ap. Sp. Science 292, 501

Chièze, J.-P., Pineau des Forêts, G., \& Flower, D.R. 1998, MNRAS, 295, 672

Ciolek, G.E. \& Roberge, W.G. 2002, Ap. J. 567, 947 
Ciolek, G.E., Roberge, W.G., \& Mouschovias, T.Ch. 2004, Ap. J. 610, 781

Draine, B.T., Roberge, W.G., \& Dalgarno, A. 1983, Ap. J. 264, 485

Flower, D.R. \& Pineau des Forêts, G. 1994, MNRAS 268, 724

Flower, D.R., Le Bourlot, J., Pineau des Forêts, G., \& Cabrit, S. 2003a, Ap. Sp. Science 287, 183

Flower, D.R., Le Bourlot, J., Pineau des Forêts, G., \& Cabrit, S. 2003b, MNRAS 341, 70

Flower, D.R. \& Pineau des Forêts, G. 2003, MNRAS 343, 390

González-Alfonso, E., Wright, C.M., Cernicharo, J., Rosenthal, D., Boonman, A.M.S., \& van Dishoeck, E.F. 2002, A\&A 386, 1074

Harwit, M., Neufeld, D.A., Melnick, G.J., \& Kaufman, M.J. 1998, Ap. J. 497, L105

Hollenbach, D. \& McKee, C.F. 1979, Ap. J. Suppl. 41, 555

Hollenbach, D. 1997, Proceedings of IAU Symposium 182, ed. B. Reipurth

Jiménez-Serra, I., Martin-Pintado, J., Rodríguez-Franco, A., \& Martín, S. 2005, Ap. J. 627, L121

Jones, A.P. 2000, J. Geophys. Res. 105, 10, 257

Le Bourlot, J. et al. 2002, MNRAS 332, 985

Lesaffre, P., Chièze, J.-P, Cabrit, S., \& Pineau des Forêts, G. 2004, A\&̛A 427, 147

Lesaffre, P., Chièze, J.-P, Cabrit, S., \& Pineau des Forêts, G. 2004, A\&A 427, 157

Liseau, R., Ceccarelli, C., Larsson, B., Nisini, B., White, G.J. et al. 1996, A\&A 315, L181

McKee, C.F. \& Hollenbach, D.J. 1980, ARAA 18, 219

Neufeld, D.A. \& Dalgarno, A. 1989, Ap. J. 340, 869

Neufeld, D.A., et al. 2000, Ap. J. 539, L107

Nisini, B., Caratti o Garatti, A., Giannini, T., \& Lorenzetti, D. 2002, A\&A 393, 1035

Nisini, B., Bacciotti, F., Giannini, T., Massi, F., Eislöffel, J., Podio, L., \& Ray, T.P. 2005, Aש্A, in press

Olofsson, A.O.H., et al. 2003, A\&A 402, L47

Pineau des Forêts, G. \& Flower, D.R. 2000, in Molecular Hydrogen in Space, eds. F. Combes \& G. Pineau des Forêts (CUP), p. 117

Smith, N., Bally, J., Shuping, R.Y., Morris, M., \& Kassis, M. 2005, A. J., in press

Snell, R.L., Hollenbach, D., Howe, J.E., Neufeld, D.A., Kaufman, M.J., Melnick, G.J., Bergin, E.A., \& Wang Z. 2005, Ap. J. 620, 758

Sternberg, A. \& Dalgarno, A. 1989, Ap. J. 338, 197

van Dishoeck, E.F. 2004, ARAA 42, 119

Viti, S., Girart, J.M., Garrod, R., Williams, D.A., \& Estalella R. 2003, A\&\&A 399, 187

Walmsley, C.M., Bachiller, R., Pineau des Forêts, G., \& Schilke, P. 2002, Ap. J. 566, L109

Wright, C.M., Drapatz, S., Timmermann, R., van der Werf, P.P., Katterloher R., \& de Graauw, T. 1996, A\&A $315, \mathrm{~L} 101$

Wright, C.M., van Dishoeck, E.F., Black, J.H., Feuchtgruber, H., Cernicharo, J., González Alfonso, E., \& de Graauw, Th. 2000, A\&A 358, 689 